\title{
Clinical Characteristics of Ischemic Colitis According to the Localization
}

\author{
Kwang Ho Kim \\ Department of Surgery, Ewha Womans University School of Medicine, Seoul, Korea
}

See Article on Page 282-286

Ischemic colitis (IC) occurs as a result of an inadequate blood supply to the colon. The segmental nature of IC can be explained by the vascular anatomy and the blood flow to the colon and the rectum. Colonic blood flow is supplied by three vessels: the superior mesentetic artery, the inferior mesenteric artery, and the superior hemorrhoidal artery. The vascular anatomy, however, is variable and often individually unique. Watershed zones are areas that are frequently susceptible to ischemic insult as a result of their location between two different vascular supplies. These areas include the splenic flexure (Griffiths' point) and the sigmoid colon (Sudeck's point), both of which may have potentially limited collateral blood flow. The significance of these anatomic points in an individual case cannot be determined without angiographic confirmation of vascular anatomy. Overall, the left part of the colon seems to be affected by IC in 75\% of the cases. The right part of the colon, including the ileocecal area, can be affected as well, but the incidence is reported to be low. However, Brandt et al. [1] reported an affection of the left colon in only $32.6 \%$ of 313 patients and more right-sided and pancolon involvement and less hepatic and splenic flexure involvement than have previous studies. Confirmed again in their study was the predominant association of certain comorbidities, i.e., hypertension, diabetes mellitus, and coronary artery disease, in patients with IC. Chronic kidney disease requiring dialysis was again a more common comorbidity in cases of right colon IC than it was for IC in general. Sepsis was a major cause of IC in patients with pancolon involvement. Chronic obstructive pulmonary disease (COPD), previous stroke, and hyper- thyroidism were shown to be the only three statistically significant independent predictors of mortality. Finally, with right colon involvement, surgery was more commonly required, and death was more frequently observed compared with the aggregate of all other patterns of IC. Carlson and Madoff [2] also suggested that the watershed zone was not especially vulnerable. Glauser et al. [3] also suggested that every segment of the colon could be affected by IC. The region of Griffith' point did not show a significant higher incidence of IC than the right colon. The right colon was significantly more often affected than the left colon in patients with non-steroidal anti-inflammatory drug (NSAID) intake. In this study, the author suggests that IC of the right colon is significantly associated with renal failure, requires more surgical treatment and shows higher mortality than IC of the left colon.

\section{REFERENCES}

1. Brandt LJ, Feuerstadt P, Blaszka MC. Anatomic patterns, patient characteristics, and clinical outcomes in ischemic colitis: a study of 313 cases supported by histology. Am J Gastroenterol 2010;105: 2245-52.

2. Carlson RM, Madoff RD. Is "ischemic" colitis ischemic? Dis Colon Rectum 2011;54:370-3.

3. Glauser PM, Wermuth P, Cathomas G, Kuhnt E, Kaser SA, Maurer CA. Ischemic colitis: clinical presentation, localization in relation to risk factors, and long-term results. World J Surg 2011;35: 2549-54.

Correspondence to: Kwang Ho Kim, M.D.

Department of Surgery, Ewha Womans University Mokdong Hospital,

911-1 Mok-dong, Yangcheon-gu, Seoul 158-710, Korea

Tel: +82-2-2650-6101, Fax: +82-2-2655-0983

E-mail: eastgate@ewha.ac.kr

(c) 2011 The Korean Society of Coloproctology

This is an open-access article distributed under the terms of the Creative Commons Attribution Non-

Commercial License (http://creativecommons.org/licenses/by-nc/3.0) which permits unrestricted non-

commercial use, distribution, and reproduction in any medium, provided the original work is properly cited. 